\title{
FUZZY C-MEANS ALGORITHM FOR SEGMENTATION OF AERIAL PHOTOGRAPHY DATA OBTAINED USING UNMANNED AERIAL VEHICLE
}

\author{
M.V. Akinin ${ }^{\mathrm{a}}$, N.V. Akinina ${ }^{\mathrm{a}}$, A.Y. Klochkov ${ }^{\mathrm{b}}$, M.B. Nikiforov ${ }^{\mathrm{c}}$, A.V. Sokolova ${ }^{\mathrm{c}}$. \\ ${ }^{a}$ Ryazan state radioengineering university, Department of Space Technology, Ryazan, Russia, 390005, Gagarina Str. 59/1 \\ ${ }^{b}$ Ryazan state radioengineering university, Ryazan, Russia, 390005, Gagarina Str. 59/1 \\ ${ }^{c}$ Ryazan state radioengineering university, Department of Electronic Computers, Ryazan, Russia, 390005, Gagarina Str. 59/1
}

KEY WORDS: fuzzy c-means, segmentation, clustering, unmanned aerial vehicle, Xie-Beni index.

\begin{abstract}
:
The report reviewed the algorithm fuzzy c-means, performs image segmentation, give an estimate of the quality of his work on the criterion of Xie-Beni, contain the results of experimental studies of the algorithm in the context of solving the problem of drawing up detailed two-dimensional maps with the use of unmanned aerial vehicles. According to the results of the experiment concluded that the possibility of applying the algorithm in problems of decoding images obtained as a result of aerial photography. The considered algorithm can significantly break the original image into a plurality of segments (clusters) in a relatively short period of time, which is achieved by modification of the original k-means algorithm to work in a fuzzy task.
\end{abstract}

\section{INTRODUCTION}

The problem of image segmentation is one of the most important steps in the process of pattern recognition.

To increase automation and quality recognition is necessary to develop segmentation algorithm, which would give high quality results in a short time. In this algorithm for the majority of applications associated with decoding of data from television cameras mounted on the drones and performing aerial photographs of the area is the algorithm of fuzzy cmeans (FCM-algorithm) based on the account properties cluster of relativity. The problem of image segmentation using FCM-algorithm reduces to identify the analyzed images of some structures of fuzzy classes, ie to finding a fuzzy partition of the original set of image pixels into classes.

\section{ALGORITHM}

\section{1 k-means}

The simplest method of splitting the image into segments is the k-means algorithm. He divides the set of elements of a vector space on a known number of clusters $k$. The action of the algorithm is that it tends to minimize the standard deviation for each cluster points. The basic idea is that at each iteration of the center of mass is recalculated for each cluster obtained in the previous step, then the vectors are divided again into clusters in accordance with which of the new centers is nearer the selected metric. The algorithm ends when at some iteration does not change clusters, in other words the difference between the new and the old position of the center is not greater than some number $\epsilon$ (parameter of the algorithm). As a measure of the distance can be selected following metrics:

- Euclidean distance, where the distance between two

${ }^{*}$ Corresponding author vectors $x_{i}$ и $x_{j}$ is calculated by the formula (1):

$$
d_{i j}=\left(\sum_{l=1}^{q}\left(x_{i l}-x_{j l}\right)^{2}\right)^{0.5}
$$

where $l$-index of the spectral characteristic, $q$ - number of spectral channels;

- Manhattan distance (urban areas) (2):

$$
d_{i j}=\sum_{l=1}^{q}\left|x_{i l}-x_{j l}\right|
$$

- Chebyshev distance - the difference between two points is defined as a maximum of one of the spectral characteristics 3;

$$
d_{i j}=\max _{l=1}^{q}\left|x_{i l}-x_{j l}\right|
$$

- power distance(4):

$$
d_{i j}=\left(\sum_{l=1}^{q}\left|x_{i l}-x_{j l}\right|^{p}\right)^{1 / r},
$$

where $r, p$ - user-defined parameters. Parameter $p$ is responsible for the gradual weighting differences on individual characteristics, the parameter $r$ is responsible for a progressive weighting of large distances between points. If both $-r$ and $p$, equal to two, then this distance coincides with the Euclidean distance.

K-means algorithm has several problems such as:

- need to know in advance the number of clusters;

- algorithm is very sensitive to the choice of the initial cluster centers:

- can not cope with the problem when the object belongs to different clusters equally or do not belong to any. 
A study was conducted disadvantages of the algorithm in order to eliminate them. For the selection of the initial cluster centers were analyzed several random number generators, as well as the calculation of the initial cluster centers so that the distance therebetween is minimized. As random number generators were investigated as follows:

- linear congruential method;

- Lagged Fibonacci generator;

- Mersenne twister.

As random number generator are encouraged to use random number generator, called «Mersenne twister» $(M$. and T., 1998). Mersenne twister has the following advantages:

- large period equal $2^{19937}-1$;

- low time costs for the generation of pseudo-random numbers and update the internal state of the random number generator;

- good statistical properties of the random number generator, as evidenced by the passage of the test DIEHARD.

With last problem of k-means well copes algorithm fuzzy c-means. Instead of a a definite answer to the question to which cluster is an object, it defines the probability that the object belongs to a particular cluster.

\subsection{Fuzzy c-means}

Suppose we have a picture $B=\left\{b_{i j}\right\} ; i=\overline{1, I} ; j=\overline{1, J}$, where $b_{i j}$ - pixel located in the $i$ th row and $j$ th column of the image. Each pixel can be represented as $b_{i j}=\left\{b_{i j k}\right\}$; $k=\overline{1, K}$, where $b_{i j k}$ - brightness of a pixel $b_{i j}$ in spectral channel $k$.

Let $C$ are fuzzy classes.

It is necessary to define a matrix $U_{\text {result }}=\left\{u_{\text {result }}{ }_{i j}\right\}$ - matrix of pixels belonging $b_{i j}$ classes $C$. Each vector $u_{\text {result }}{ }_{i j}=\left\{u_{\text {result }}{ }_{i j c}\right\} ; u_{\text {result }}{ }_{i j c} \in[0,1] ; c=\overline{1, C}$ describes the degree of membership pixel $b_{i j}$ classes $C$.

Each class $c$ is the central vector $v_{c}$, which form the set of centers of classes $V$.

There is also the objective function $T(U, V)$ (5):

$$
T(U, V)=\sum_{i j} \sum_{c=1}^{C} u_{i j c}^{m} d^{2}\left(b_{i j}, v_{c}\right),
$$

where $d$ - Euclidean distance, $m$ - fuzzifikator.

Target FCM-algorithm reduces actually minimizing $T(U, V)$

FCM-parameters of the algorithm are:

- the number of classes $C ; C \in \mathrm{N} ; C>1$;

- the maximum number of iterations of the algorithm $N_{\max } ; N_{\max } \in \mathrm{N}$
- parameter convergence of the algorithm $\epsilon ; \epsilon \in \mathrm{R}$;

- fazzifikator $m$.

FCM-algorithm (Bezdek et al., 1984) segmentation consists of the following steps.

1. As the current fuzzy partition of $U$ randomly given a fuzzy partition on $C$ non-empty clusters, which are described by membership functions $F=\left\{f_{c}\right\} ; f_{c}\left(b_{i j}\right)$; $c=\overline{1, C}$.

2. For the current fuzzy partition of $U$ is calculated coordinates of the centers of classes $v_{c}(7)$, forming the set $V ;|V|=C$, and the objective function value $T(U, V)$ :

$$
\begin{gathered}
v_{c}=\left\{v_{c k}\right\} ; \\
v_{c k}=\frac{\sum_{i j} b_{i j k} u_{i j c}^{m}}{\sum_{i j} u_{i j c}^{m}} ;
\end{gathered}
$$

3. If the condition (8) or condition (9), then as $U_{\text {result }}$ takes $U$ and the algorithm stops.

$$
\begin{gathered}
\left|T(U, V)-T_{\text {old }}\right| \leq \epsilon ; \\
n<N_{\max }
\end{gathered}
$$

where $T_{\text {old }}$ - value of the objective function at the previous iteration of the algorithm, $n$ - number of the current iteration.

4. Formed fuzzy partition of $U_{\text {next }}$ to $B$ on $C$ non-empty clusters, which are described by membership functions $F$, according to the formula (10):

$$
u_{i j c}=f_{c}\left(b_{i j}\right)=\frac{1}{\sum_{\xi=1}^{C}\left(\frac{d\left(v_{c}, b_{i j}\right)}{d\left(v_{\xi}, b_{i j}\right)}\right)^{\frac{2}{m-1}}} .
$$

5. As $U$ is taken $U_{\text {next }}$.

6. Proceed to the step 2 algorithm.

Final classification of image pixels $B$ is that each pixel belongs to the class $q_{i j}$ such that $q_{i j}=\arg _{c} \max u_{\text {result } i j c}$.

According to the results of the classification of the image $B$ of the segment - a separate segment is a connected set of pixels belonging to the same class.

Normally, as an indicator of the quality of fuzzy clustering is used objective function (5) FCM-algorithm, the value of which should be minimized. In addition, there are other indicators of the quality. One of the well-established indicators of quality of clustering is Xie-Beni index (11) (D.Dumitrescu et al., 2000):

$$
X B=\frac{\sum_{i j} \sum_{c=1}^{C} u_{i j c}^{m} d^{2}\left(b_{i j}, v_{c}\right)}{n * \min _{t \neq c} d^{2}\left(v_{c}, v_{t}\right),} \rightarrow \min
$$

where $n$ - the number of pixels in the image.

Xie-Beni index takes into account the fuzzy membership degree of image points center of the cluster, as well as the 
geometric arrangement of cluster centers and image points, and is the most informative indicator of the quality of clustering in the evaluation of compactness and good separation of the clusters. As practice shows, with good results of fuzzy clustering $X B<1$. At the same time as the desired number of clusters is advisable to select the one for which the index takes the minimum value.

\section{EXPERIMENT}

Experimental studies of the proposed algorithm were carried out in the context of a two-dimensional drawing up detailed maps of the area on the data obtained from the television cameras mounted on unmanned aircraft helicopter type (geksakopter).

To carry out aerial photography in the visible range of frequencies used UAV helicopter type, equipped with a television (TV) camera and a set of navigation equipment. The experiment was conducted in the computer system, having the following features: the CPU - Intel Core i5 3337U (the CPU operating frequency is $2.7 \mathrm{GHz} ; 4$ computer kernels; the cache capacity L3 is $3 \mathrm{MB}$ ), the RAM is $4 \mathrm{~GB}$ DDR3, OS GNU / Linux (kernel version Linux: 3.8.6).

For the experiment using geksakoptera received a number of video sequences with different characteristic objects such as bodies of water, dirt roads, technical facilities.

Produced a study to determine the number of clusters on a particular image, the optimal number of clusters was determined by v-fold cross-validation (Murty et al., 2012), and given the Xie-Beni index. For scenes represented the number of clusters was 5-10.

The analysis of the initial determination of cluster centers. Compared with a random distribution, was the best allocation of $C$ outermost points (the first two points are allocated to the maximum pairwise distances, each should be chosen so that the distance from it to the nearest already been selected as possible).

According to the results of experimental studies considered algorithm showed high quality and relatively low time costs. The results of the algorithm are presented in figures 1 and 2 .

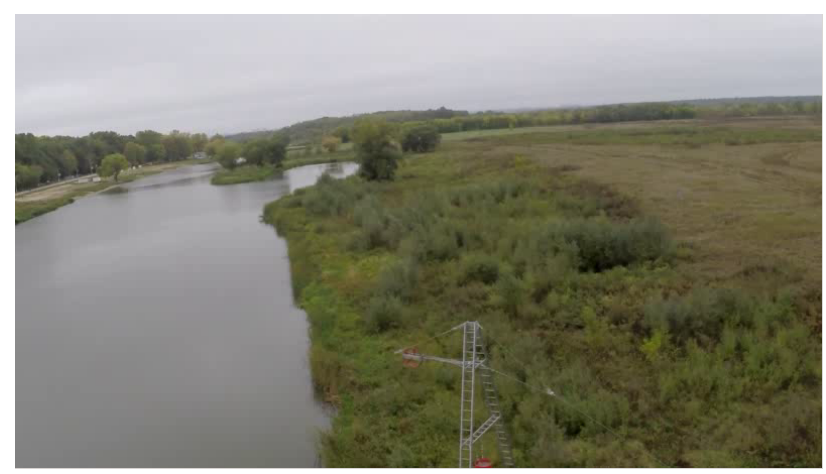

Figure 1: Original image

\section{CONCLUSIONS}

Considered segmentation algorithm lends itself to parallelization, so along with the high levels of quality (compared

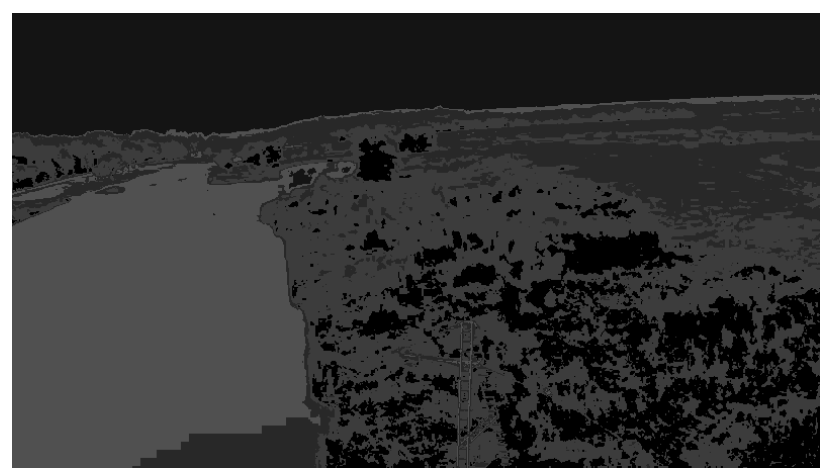

Figure 2: FCM-clustering

to the standard algorithm k-means) showed good temporary results. Considered segmentation algorithm can be used in problems of combining digital maps and virtual terrain model using pattern recognition, as well as for the construction of high detailed two-dimensional and three-dimensional maps and terrain models.

\section{REFERENCES}

Bezdek, J., Enrlich, R. and Full, W., 1984. Fcm: the fuzzy c-means clustering algorithm. Computers and Geosciences 10(2-3), pp. 191-203.

D.Dumitrescu, B.Lazzerini and L.C.Jain, 2000. Fuzzy sets and their application to clustering and training. CRC Press LLC, Florida.

M., M. and T., N., 1998. Mersenne twister: a 623dimensionally equidistributed uniform pseudo-random number generator. ACM Transactions on Modeling and Computer Simulation (TOMACS; volume 8, issue 1) pp. $3-30$.

Murty, G. S., Kumar, D. V. V. and Naresh, T., 2012. Learning number of clusters in unlabeled dataset using rotation estimation. International journal of engineering research and applications 2, pp. 41-45. 